\title{
SÍNDROME DA ARTÉRIA MESENTÉRICA SUPERIOR COM ESTENOSE ARTERIAL MESENTÉRICA SUPERIOR CRÔNICA COMO COMPLICAÇÃO TARDIA DE COLECISTECTOMIA
}

\section{SUPERIOR MESENTERIC ARTERY SYNDROME WITH CHRONIC SUPERIOR MESENTERIC ARTERIAL STENOSIS AS THE LATE COMPLICATION OF CHOLECYSTECTOMY}

Atsuo Nakanishi,TCBC-PA 1,2 ; Hudson Fernandes Barile3; Renan Rocha Granato ${ }^{1,3}$; Anísio Fernando Eduardo Silva Vieira Chaves ${ }^{4}$; Evellyn Vitória Sousa de Loureiro ${ }^{1}$.

\section{RESUMO}

A Síndrome da Artéria Mesentérica Superior ou de Wilkie é uma causa rara de obstrução duodenal e resulta da compressão da terceira porção do duodeno pela artéria mesentérica superior e pela artéria aorta. É apresentado o caso de uma mulher de 49 anos de idade, com sintomatologia de dor e distensão pós-prandial e perda ponderal com doze meses de evolução após a colecistectomia. Após investigação e exclusão de outras patologias, é diagnosticada esta síndrome associada à estenose crítica oclusão arterial crônica da Artéria Mesentérica Superior. O caso demonstra o quadro clínico, o estudo diagnóstico e as opções terapêuticas.

Descritores: Síndrome da Artéria Mesentérica Superior. Síndrome de Wilkie. Obstrução Duodenal. Oclusão Arterial Mesentérica Crônica. Colecistectomia.

\section{ABSTRACT}

Superior Mesenteric Artery or Wilkie Syndrome is a rare cause of duodenal obstruction and results from the compression of the third portion of the duodenum by the superior mesenteric artery and the aorta. The case of a 49-year-old woman is presented, with symptoms of pain and postprandial distension and weight loss after twelve months of evolution after cholecystectomy. After investigation and exclusion of other pathologies, this syndrome associated with chronic critical stenosis arterial occlusion of the Upper Mesenteric Artery is diagnosed. The case demonstrates the clinical feature, the diagnostic study and the therapeutic options.

Keywords: Superior Mesenteric Artery Syndrome. Wilkie Syndrome. Duodenal Obstruction. Chronic Mesenteric Arterial Occlusion. Cholecystectomy.

\section{INTRODUÇÃO}

A Síndrome da Artéria Mesentérica Superior (SAMS) ou Sindrome de Wilkie é uma afecção rara, com prevalência na população geral de $0,013 \%$ a $0,3 \%$, sendo mais frequente em mulheres jovens, entre 20 e 40 anos de idade ${ }^{1}$. Caracteriza-se pela obstrução da terceira porção do duodeno pela artéria mesentérica superior, anteriormente, e pela aorta e coluna vertebral, posteriormente ${ }^{1-6}$.

A causa mais frequente dessa patologia é a perda ponderal significativa ocasionada por doenças agudas ou crônicas. O quadro clínico é composto por dor e distensão abdominal pós-prandial, náuseas, vômitos e emagrecimento acentuado ${ }^{2}$.
O diagnóstico é realizado com exames radiológicos contrastados. A terapêutica é, inicialmente, conservadora e, nos casos refratários, o tratamento cirúrgico passa a ser recomendado ${ }^{3}$. A duodenojejunostomia é a técnica de eleição ${ }^{4}$.

A seguir, é apresentado o quadro clínico, o estudo diagnóstico e a terapêutica da Síndrome de Wilkie em uma mulher de 49 anos de idade, com sintomatologia de dor e distensão pós-prandial e perda ponderal com doze meses de evolução.

\section{RELATO DO CASO}

Paciente do sexo feminino, 49 anos, tabagista há 35 anos, sem comorbidades prévias foi submetida à colecistectomia la-

\footnotetext{
${ }^{1}$ Universidade Federal do Pará, Faculdade de Medicina - Altamira - PA - Brasil

${ }^{2}$ Clínica Gastroendo, Serviço de Gastroenterologia e Endoscopia - Altamira - PA - Brasil

${ }^{3}$ Hospital Municipal de Altamira, Serviço de Cirurgia - Altamira - PA - Brasil

${ }^{4}$ Clínica MaxxiSaúde, Serviço de Radiologia - Altamira - PA - Brasil
} 
Nakashini et al.

Sindrome da artéria mesentérica superior com estenose arterial mesentérica superior crônica como complicação tardia de colecistectomia

Relato de caso

parotômica devido à colelitíase em julho de 2019 , sem relato de intercorrência operatória. No $15^{\circ}$ dia pós-operatório, houve início insidioso de epigastralgia e dispepsia pósprandial com piora progressiva, evoluindo com náuseas, vômitos e perda ponderal acentuada, tendo emagrecido $14 \mathrm{~kg}$ nos 12 meses de evolução.

Foi realizada endoscopia digestiva alta com diagnóstico de gastrite com metaplasia intestinal. Raio-X contrastado de esôfago-estômago-duodeno não evidenciou claramente a obstrução duodenal permitindo a passagem de contraste liquido. A tomografia computadorizada de abdome superior com contraste venoso mostrou um ângulo aórtico-mesentérico de $15^{\circ}$, caracterizando a Sindrome de Wilkie, a suboclusão junto à $4^{\circ}$ porção duodenal com dilatação da $3^{\circ}$ porção duodenal, e a estenose crítica oclusão da emergência da artéria mesentérica superior e do tronco celiaco, de natureza ateromatosa, porém observou-se que já há enchimento de contraste nos seus territórios.



Figura 1. Tomografia abdominal com contraste demonstrando ângulo aorto-mesentérico de $15^{\circ}$ e estenose crítica oclusão das origens da Artéria Mesentérica Superior e do tronco celíaco.

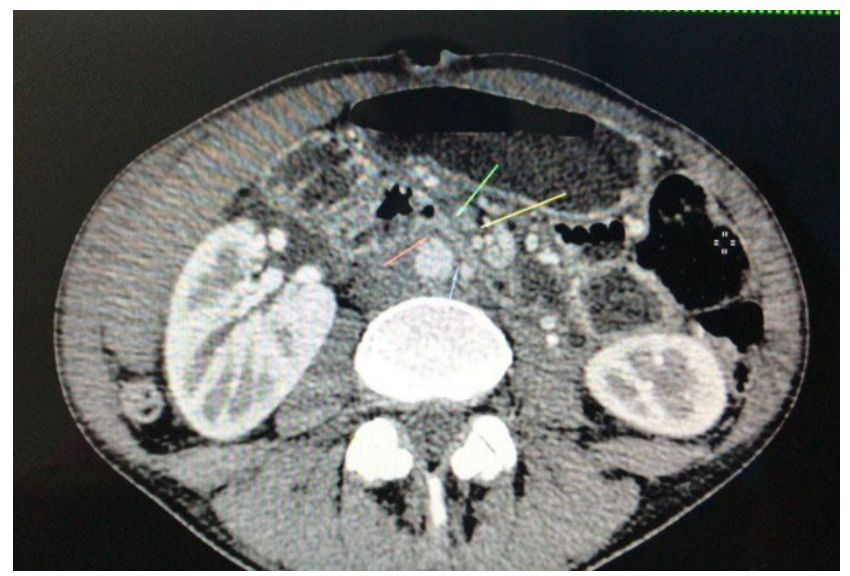

Figura 2. Tomografia abdominal com contraste demonstrando a suboclusão junto à $4^{\circ}$ porção duodenal com dilatação da $3^{\circ}$ porção duodenal.
Foi iniciado tratamento medicamentoso, dietético e postural pós-prandial por 3 meses, sem melhora significativa. Optou-se, então, por tratamento cirúrgico e foi submetida em julho de 2020. Durante a cirurgia, foi observada uma aderência firme do ângulo duodenal superior ao leito vesicular do figado, com afiação do referido ângulo entre bulbo e $2^{\circ}$ porção duodenal com provável obstáculo da passagem de bolo alimentar, dilatação significativa da $3^{\circ}$ porção duodenal, e dilatação das artérias cólicas esquerda e média, além da visualização da Arcada de Riolan. Foi realizada a duodenojejunostomia látero-lateral entre ângulo duodenal inferior e jejuno proximal com sutura contínua de Albert-Lambert, posicionamento da sonda nasoenteral Dobbhoff 12FR para nutrição enteral precoce pósoperatória, e colocação de dreno ao redor da anastomose. O procedimento demorou cerca de 120 minutos devido à presença de extensa aderência sem intercorrências.

A evolução pós-operatória foi satisfatória, sendo administrada enoxaparina e antiagregante plaquetário, tendo a alta hospitalar no $10^{\circ}$ dia pós-operatório, com a prescrição de apenas antiagregante plaquetário, cilostazol na dose de $200 \mathrm{mg} /$ dia.

Foi realizada a reavaliação clínica e endoscópica no $30^{\circ}$ dia pós-operatório, com melhora significativa com desaparecimento do quadro de epigastralgia e vômitos, com ganho ponderal de $2 \mathrm{~kg}$, persistindo apenas certa dispepsia pós-prandial que está relacionada a angina mesentérica devido à estenose crônica da Artéria Mesentérica Superior, porém os efeitos farmacológicos vasodilatador e antiagregante plaquetário do cilostazol reduziram a intensidade da dispepsia pós-prandial. A imagem endoscópica da duodenojejunostomia mostrou uma anastomose bem ampla sem alterações macroscópicas

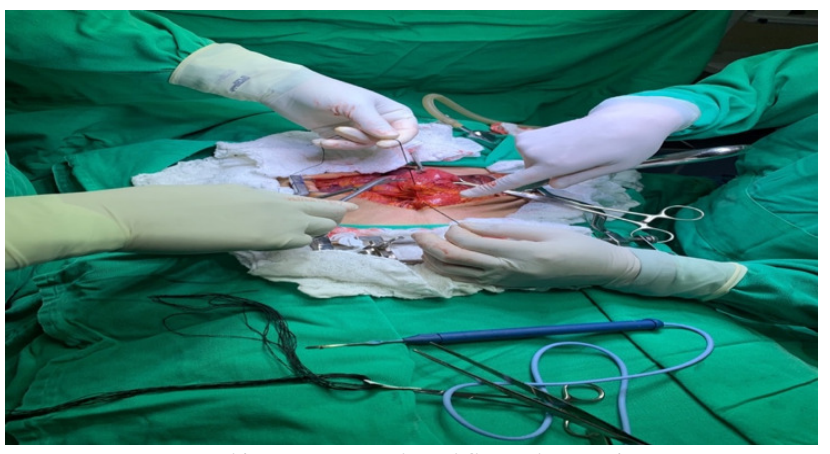

Figura 3. Dilatação significativa da $3^{\circ}$ porção duodenal. 


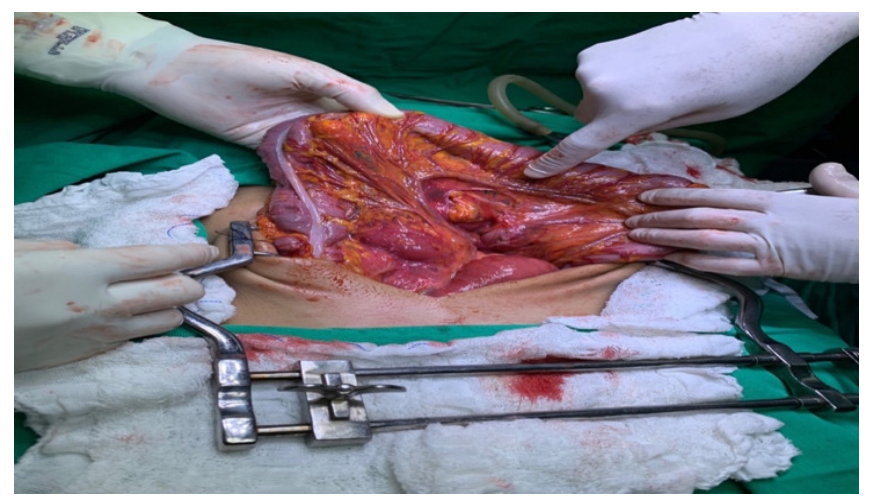

Figura 4. Arcada de Riolan.

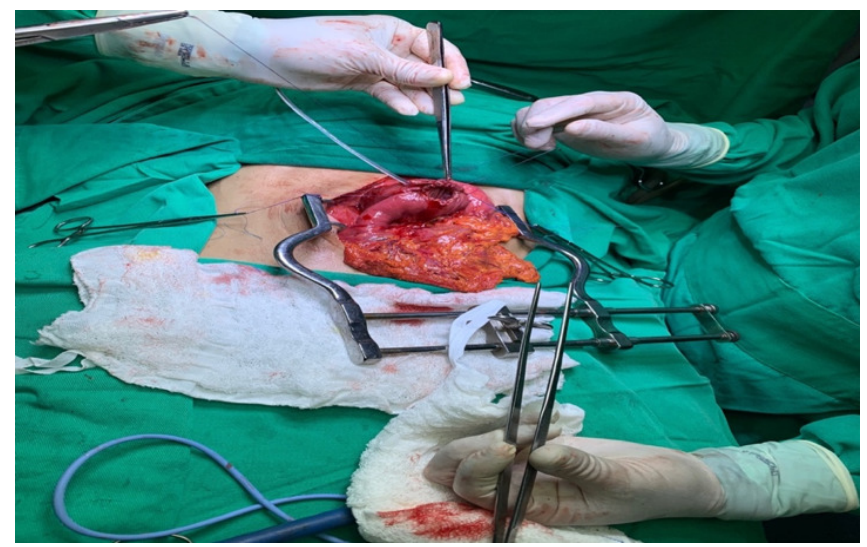

Figura 5. Duodenojejunostomia látero-lateral em confecção.

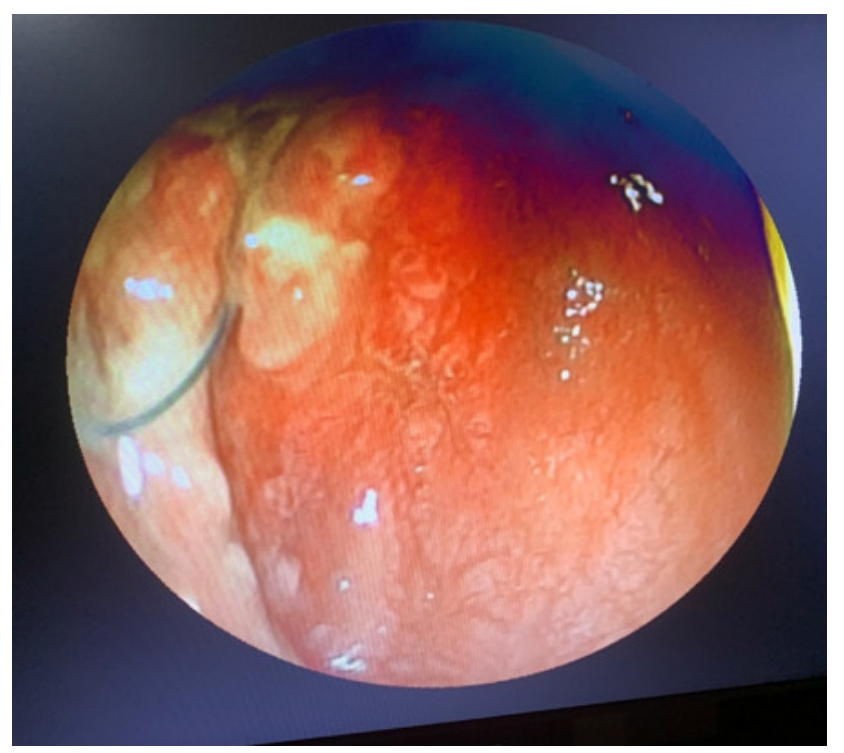

Figura 6. Aspecto endoscópico pós-operatório da duodenojejunostomia.

\section{DISCUSSÃO}

A Sindrome da Artéria Mesentérica Superior (SAMS) foi descrita pela primeira vez em 1861, porém, a patologia foi caracterizada apenas em 1920, por Wilkie ${ }^{4}$. É uma doença pouco frequente, com apenas 500 casos descritos na literatura recente 4 . Nesse contexto, a SAMS associada a estenose oclusão arterial crônica do tronco celí- aco e da Artéria Mesentérica Superior é ainda mais rara e, até o momento, nunca foi relatada. A SAMS pode ser adquirida ou congênita. As etiologias mais frequentes resultam de perda ponderal significativa secundária a outras patologias ${ }^{2,5}$. Dessa maneira, ocorre a depleção da gordura perimesentérica e retroperitoneal, diminuindo o ângulo aorto-mesentérico ${ }^{1}$. Provável causa da perda ponderal acentuada no nosso caso foi a aderência firme supracitada com obstáculo da passagem de bolo alimentar para $2^{\circ}$ porção duodenal com vômitos frequentes. Normalmente o ângulo formado pela aorta abdominal e pela artéria mesentérica superior está entre 20 e $70^{\circ} 8$. Quando esse ângulo é menor que $20^{\circ}$, como no caso apresentado, há compressão mecânica do duodeno, o que causa sintomas de obstrução intestinal crônica.

A sintomatologia é variável e inespecífica. Manifesta-se, geralmente, por dor abdominal ou enfartamento pós-prandial, náuseas e vômitos biliares, saciedade precoce, anorexia e perda ponderal2,8. O diagnóstico é dificil, pois implica um elevado indice de suspeição. A investigação diagnóstica com exames complementares é iniciada com exames radiológicos contrasta$\mathrm{dos}^{4}$. A tomografia computorizada com contraste é o exame de eleição, pois não é invasivo e fornece detalhes como a posição da artéria mesentérica superior e o seu ângulo com a aorta, assim como permite excluir outras causas de obstrução duodenal4. Quando as radiografias contrastadas não forem suficientes para a realização do diagnóstico, exames invasivos, como arteriografia e angioressonância, podem ser solicita$\operatorname{dos}^{8}$.

Confirmado a Sindrome de Wilkie, a primeira abordagem deve ser clínica, por meio de suporte nutricional, fracionamento da dieta e manobras posicionais após a ingestão alimentar1,5. As manobras que podem ser utilizadas são decúbito lateral esquerdo, genupeitoral ou flexão anterior do corpo, uma vez que essas posições promovem maior amplitude do ângulo aortomesentérico ${ }^{4}$.

No entanto, cerca de $50-70 \%$ dos casos necessitam de tratamento cirúrgico. Nesse sentido, existem algumas alternativas que podem ser utilizadas com a técnica aberta ou minimamente invasiva, como o procedimento de Strong, a duodenojejunos- 
tomia ou a gastrojejunostomia ${ }^{4,7}$. O procedimento de Strong, mais utilizado em crianças e adolescentes, apresenta índice de sucesso de cerca de $75 \%$, e a gastrojejunostomia confere maior risco de aparecimento de úlceras pépticas. Portanto, a duodenojejunostomia látero-lateral é a técnica de escolha, com indice de sucesso de $90 \% 4,5$, razão pelo qual foi o procedimento de escolha nesse caso.

Como mencionado, a paciente apresentou, associado à Sindrome de Wilkie, estenose crítica oclusão arterial crônica da Artéria Mesentérica Superior e do Tronco celíaco.

A isquemia mesentérica crônica ocorre quando há oclusão de pelo menos dois, dos três vasos que suprem o intestino: Tronco celiaco, Artéria Mesentérica Superior (AMS) e Artéria Mesentérica Inferior (AMI). O principal agente é o processo aterosclerótico, que responde por $90 \%$ dos ca$\operatorname{sos}^{10}$.

O quadro clínico é caracterizado por dor abdominal pós-prandial, emagrecimento e alterações no trânsito intestinal, o que confunde com a sintomatologia da SAMS. O diagnóstico definitivo é realizado com arteriografia. Mas outros exames podem realizar o diagnóstico, como angiotomografia, angiorressonância e ultrassonografia com doppler 9 . O tratamento pode ser clínico, cirúrgico ou endovascular' .

Optou-se pelo tratamento medicamentoso com antiagregante plaquetário. Pois, há uma extensa rede de vasos sanguineos colaterais que protegem o intestino em períodos de perfusão tecidual inadequada. Nesse sentido, o tronco celíaco e a AMS comunicam-se principalmente por meio das artérias pancreaticoduodenais superiores e inferiores. A AMS e a AMI comunicam-se através da arcada Marginal de Drummond e da arcada de Riolan ${ }^{10}$.

Por fim, o caso relatado é relevante, pois demonstra que o diagnóstico preciso e a terapia adequada corroboram para melhores resultados, assim como por descrever o primeiro caso, dentro do nosso alcance da pesquisa bibliográfica, da associação da Sindrome da Artéria Mesentérica Superior com estenose oclusão arterial mesentérica superior crônica.

\section{REFERÊNCIAS}

1. Khodear Y, Al-Ramli W, Bodnar Z. Laparoscopic management of a complicated case of Wilkie's syndrome: a case report. Int $\mathrm{J}$ Surg Case Rep. 2017;37:177-9.

2. Farina R, Foti PV, Cocuzza G, Costanzo V, Costanzo G, Conti A, et al. Wilkie's syndrome. J Ultrasound. 2017;20(4):339-42.

3. Gebhart T. Superior mesenteric artery syndrome. Gastroenterol Nurs. 2015;38:189-93.

4. Silva E, Ribeiro C, Guerreiro S, Domingues A. Sindrome de Wilkie: a propósito de um caso clínico. Rev Port Cir. 2016;37:25-8.

5. Barquín Yagüez J, Abadía Barnó P, García Pérez JC. Superior mesenteric artery syndrome: an uncommon cause of intestinal obstruction. Rev Esp Enferm Dig. 2017;109(1):60.

6. Aneiros Castro B, Cano Novillo I, García Vázquez A, Martín Alelu R, Gómez Fraile A. Wilkie's syndrome in monozygotic twins treated by 3-D laparoscopic duodenojejunostomy. Asian J Endosc Surg. 2019;12(1):125-7.

7. Konstantinidis H, Charisis C, Kottos P. Robotic Strong's procedure for the treatment of superior mesenteric artery syndrome. Description of surgical technique on occasion of the first reported case in the literature. Int $\mathrm{J}$ Med Robot. 2018;14(1):1876.

8. Cesconetto D, Mattosinho T, Gestic M, Chaim A. Síndrome da artéria mesentérica superior: relato de caso. GED Gastroenterol Endosc Dig. 2012;31(4): 138-41.

9. Ferreira R, Canedo A. Isquemia Mesentérica Crônica. Galicia Clin. 2013; 74(4):195.

10. Filgueiras DTB, Sampaio FC, Silva SGJ, Silva MAM, Cardoso RS. Tratamento da Isquemia Mesentérica Crônica através da Angioplastia do Tronco Celíaco. Revista Ciências em Saúde. 2016;6(3):142-51.

\section{Endereço para correspondência:}

Atsuo Nakanishi

E-mail: atsuonakanishi@hotmail.com atsuo@ufpa.br 